\title{
„Raabe im Sterben.“ \\ Bemerkungen anlässlich einer Notiz Franz Kafkas*
}

,-- und so ist das, was ihr meine sonnige Heiterkeit nennt, nichts als das Atemschöpfen eines dem Ertrinken Nahen." ${ }^{11}$

Von dem Todesfall, der sich am 15. November 1910 in Braunschweig zutrug, weiß der nachmals vielleicht berühmteste literarische Zeitgenosse rückblickend nur eine winzige Szene zu berichten. Zwischen dem 20. und 22. Dezember 1921 hält Kafka in seinem Tagebuch unter anderem die folgenden Bemerkungen fest:

Vieles durchgelitten in Gedanken

[...]

Besser zu durchdenken:

Raabe im Sterben, als ihm seine Frau über die Stirn strich: Das ist schön ${ }^{2}$

Eine an Beiläufigkeit nicht zu übertreffende Notiz, könnte man denken; stünde sie nicht in Kafkas Tagebuch, würde man sie selbst an einem so feierlichen Gedenktag schwerlich noch zitieren. Sie würde wohl auch nicht wesentlich interessanter durch den Umstand, dass Kafka - der sonst an Raabe nirgends Interesse zeigt und dessen Lektüre hier deshalb etwas überraschend erscheint - sie aus dem kurz zuvor erschienenen Raabe-Gedenkbuch übernommen hat, in dem der Mitherausgeber Hans Martin Schultz unter der Überschrift Der alte Herr ,persönliche Erinnerungen“" an den alten Raabe sammelte, darunter auch an die letzten Augenblicke seines Lebens:

Er starb mit sich und der Welt vollkommen in Frieden. [...] Als ihm seine treue Lebensgefährtin mit der Hand über die Stirn strich, sagte er noch: „Das ist schön.“3

* Festvortrag im Rahmen des Festaktes zu Wilhelm Raabes 100. Todestag in der Dornse des Braunschweiger Altstadtrathauses am 24. September 2010.

1 Notiz vom 12. Januar 1878. Zitiert nach Wilhelm Raabe: Einfälle. Aphorismen in zeitlicher Folge. In: Ders.: Werke in Einzelausgaben, Bd. 10: Altershausen. Hg. von Hans-Jürgen Schrader, Frankfurt/Main 1985, S. 186.

2 Franz Kafka: Tagebücher. Hg. von Hans-Gerd Koch, Michael Müller und Malcolm Pasley, Frankfurt/Main 1990, S. 876.

3 Raabe-Gedenkbuch. Im Auftrage der Gesellschaft der Freunde Wilhelm Raabes zum 90. Geburtstage des Dichters hg. von Constantin Bauer und Hans Martin Schultz, Berlin 1921, S. 141-148: „Der alte Herr. Persönliche Erinnerungen von Hans Martin Schultz“. Ebenfalls in Wilhelm Raabe: Sämtliche Werke. 20 Bde. und 5 Ergänzungsbände. Hg. im Auftrag der Braunschweigischen Wissenschaftlichen Gesellschaft von Karl Hoppe und Jost Schillemeit, Göttingen 1960ff., Erg.-Bd. 4: Gespräche. Ein Lebensbild in Aufzeichnungen und Erinnerungen der Zeitgenossen. Hg. von Rosemarie Schillemeit, Göttingen 1983, S. 316. Nach den jeweils neuesten Bandauflagen dieser Braunschweiger Ausgabe 
Vielleicht hätten wir uns ohne Kafka gar nicht mehr an diese Szene erinnert; ${ }^{4}$ zu einer rührenden Anekdote taugt sie ja auch wirklich nicht, dazu bleibt sie zu alltäglich. Raabes Biographen jedenfalls haben sie nicht beachtet; selbst der sonst auf jedes Detail aufmerksame Werner Fuld zitiert zwar den apokryphen Satz, in dem der Sterbende sich wie einen Fremden beobachtet: „Ist er noch nicht tot?“5 Aber die kleine Geste, die Kafka als einzige in sein Notizbuch einträgt, übergeht er. Natürlich, verständlich - denn versteht es sich nicht von selbst, dass ein Kranker oder gar Sterbender eine solche Geste eben „schön“ findet?

Das einzige Ziel dieses Fest- und Gedenkvortrags ist es, Ihnen Kafkas Fixierung und Bewertung dieser Szene plausibel zu machen. Dazu muss ich allerdings weit ausholen und dem mir von ihr vorgegebenen Stichwort „Raabe im Sterben" einige Aufmerksamkeit widmen. Erst abschließend werde ich dann auf diese Notiz zurückkommen. Ich habe also zu dieser Veranstaltung keine Forschungsergebnisse vorzustellen, keine überraschenden Thesen zu erörtern, keine neuen Funde zu präsentieren. Vielmehr nehme ich Raabes 100. Todestag zum Anlass, über einige rote Fäden nachzudenken, die mir im Lichte von Kafkas Notiz (und beim Rückblick auf eine jahrzehntelange Beschäftigung mit diesem Autor) sichtbar werden. ${ }^{6}$ Zunächst skizziere ich dazu sehr knapp Tod und Sterben als Epochenthemen von Raabes Lebens- und Schreibzeit; dann wende ich mich Raabes eigenen Todes- und Sterbensbildern zu und frage nach den moralischen Folgerungen, die er daraus ableitet, ehe ich wieder am Ausgangspunkt ankomme. Das Ganze bildet einen kleinen Versuch über Raabe'sche Humanität - es wird also, trotz der Überschrift und einer eher anlassgemäß düsteren ersten Hälfte, am Ende womöglich doch noch ein tröstliches Unterfangen.

wird im Folgenden unter Verwendung der Sigle BA (mit Bandnummer und Seitenzahl) zitiert. - Mit Kafkas Rezeption dieser Quelle (und dieser selbst) habe ich mich zuerst in einer Miszelle beschäftigt, auf die ich hier zurückgreife: Heinrich Detering: „Raabe im Sterben“. Zu einer nachgelassenen Erzählung Franz Kafkas. In: Mitteilungen der RaabeGesellschaft 73 (1986), H. 2, S. 17-20.

4 Von der übrigens Klara Behrens-Raabe eine durchaus abweichende Version erzählt (BA Erg.-Bd. 4, S. 317).

5 Werner Fuld: Wilhelm Raabe. Eine Biographie, München und Wien 1993, S. 363, zitiert nach der Erinnerung von Paul Wasserfall: Vom Hause Raabe. In: Raabe-Gedenkbuch (wie Anm. 3), S. 3-22, hier S. 22 (auch BA Erg.-Bd. 4, S. 317).

6 Dabei beziehe ich mich auf zahlreiche Erträge der neueren Raabe-Forschung, deren vollständige Nennung auf eine Bibliographie der letzten zwanzig Jahrgänge des Jahrbuchs der Raabe-Gesellschaft [im Folgenden: JbRG] sowie der darin rezensierten Forschungsbeiträge hinausliefe. Um das zu vermeiden und den Charakter des Festvortrags auch in der Druckfassung zu wahren, verweise ich nur auf jeweils unmittelbar einschlägige Forschungsbeiträge. 
I.

„Raabe im Sterben“: Kafkas Wendung ist zunächst so wörtlich wie möglich zu nehmen. Denn umfassend und ausweglos findet sich der „Meister Autor“ Raabe (so wie ihn jüngst Alois Brandstetters Gemälde zeigt) ${ }^{7}$ von Beginn an vor in einer Welt des Sterbens. Das muss erläutert werden.

Keine Epoche der deutschen Literatur seit dem Barock, seit der Zeit also von Opitz' Trostgetichte in Widerwerttigkeit des Krieges und Gryphius' KirchhofsGedanken, ist in solchem Grade von der Erfahrung und von Vorstellungen des Sterbens besessen gewesen wie das 19. Jahrhundert nach dem Tode Goethes, wie dann in der zweiten Hälfte dieses Jahrhunderts vor allem die zweite Generation des Poetischen Realismus. Und ich meine damit nicht nur allgemein irgendeinen Pessimismus oder Nihilismus, sondern eine buchstäbliche Obsession durch Bilder und Begriffe, Szenen oder Metaphern des Sterbens. ${ }^{8}$

Bilder und Begriffe von Sterben und Tod artikulieren die verborgene Rückseite jenes Fortschrittsoptimismus, der das ökonomische und politische Deutschland des 19. Jahrhunderts so lautstark dominiert, dass bereits diese Lautstärke etwas Misstrauenerweckendes hat. Denn es scheint ja mit einem nie dagewesenen Elan aufwärts zu gehen in Industrie und Technik, in der Wirtschaft, in der Medizin und den Naturwissenschaften, aber auch in den mit wachsendem Eifer diesem Vorbild folgenden historischen Wissenschaften und Philologien und in den entsprechenden philosophischen Strömungen von den sehr unterschiedlichen Varianten der post-hegelianischen Geschichtsphilosophie bis zu den post-darwinistischen Amalgamierungen von Evolutionstheorie und Religion in Bewegungen wie Haeckels Monismus. Wilhelms II. Satz, er führe die Deutschen herrlichen Zeiten entgegen, spricht 1888 aus, was als gleichsam staatsoffizielle Tendenz eines staatstragenden Besitzbürgertums das Jahrhundert begleitet hat. (Es ist das Jahr, in dem Raabe Das Odfeld veröffentlicht.) Im florierenden Hochkapitalismus, dieser Aufstiegs- und Triumphgeschichte der bürgerlichen Ökonomie - die sich doch heimlich schon als unterminiert wahrnehmen muss durch mit knapper Not überlebte Börsencrashs, durch Kriegsängste und Weltkriegsahnungen, durch aufflackernde soziale Unruhen -, in dieser bürgerlichen Ökonomie sind es die bürgerlichen Intellektuellen, in deren Texten die Verlust- und Gegenrechnungen aufgemacht werden.

7 Alois Brandstetter: Raabe, der Alte. In: JbRG 2010, S. 1f. Dazu Hans-Jürgen Schrader: Der Raabe-Preisträger Alois Brandstetter als Maler. Anlässlich seines Raabe-Porträts mit Totenkopf. In: Ebd., S. 3-13.

8 Ausführlich und in kulturwissenschaftlicher wie literaturgeschichtlicher Kontextualisierung wird dieses Thema jetzt in einer demnächst erscheinenden Habilitationsschrift erörtert, auf die ich hier nur nachdrücklich hinweisen kann: Andreas Blödorn: Todessemantiken. Sinneswahrnehmung und Narration im Wandel literarischer Epistemologie des späten 19. Jahrhunderts. Habilitationsschrift Bergische Universität Wuppertal 2010 (mit umfangreichen Hinweisen auch auf weitere Forschungsliteratur). 
Aber nicht nur in der sozialen und ökonomischen Welt lag das Sterben in der Luft. Ob sie nicht überall den Verwesungsgeruch des toten Gottes wahrnähmen, fragt Nietzsches ,toller Mensch“ 1882 seine entsetzten Zuhörer. ${ }^{9}$ Das Sterben diente ihm und anderen als Metapher für das Ende der metaphysischen Gewissheiten, deren vorerst letzte die Hegel'sche und daraufhin die Marx'sche Geschichtsphilosophie gewesen waren; und immer weiter in die elementaren Gewissheiten ging die Erschütterung - zuerst bis in die Grundfesten des Ich, das doch, von Descartes bis zur romantischen Subjektphilosophie, als ineffabile gegolten hatte und von dem Nietzsche nun verkündete, es sei ,zum Wortspiel“ geworden, ${ }^{10}$ nur ein Pronomen im Sprachspiel einer trügerischen Syntax, und von dem Freud wenig später, noch während Raabe an seinen letzten Romanen arbeitet, erklären wird, es sei nicht mehr „Herr im eigenen Haus“; ;1 dann bis in die Grundfesten der Sprache selbst. Was er als „Tod Gottes“ nicht eigentlich proklamiert, sondern diagnostiziert habe, das, so macht Nietzsche seinen voreiligen Anhängern klar, sei nur der Anfang eines unaufhaltsamen Zerfalls, der auch die traditionellen Vorstellungen von Subjekt und Erkenntnis, weltanschaulichen, epistemologischen und moralischen Gewissheiten erfassen werde. Der Schlussstein war aus dem Gewölbe genommen, und nun blieb kein Stein auf dem anderen; was der Einsturz unter sich begrub, war eine jahrhundertlange Geschichte des metaphysischen und dann eines durchaus weltlichen Optimismus; das Sterben wurde zu einer heimlichen Leitmetapher der Epoche.

Auch die Anfänge der literarischen Bewegung, in die Raabes Werk sich von Beginn an einfügt, hatten im Zeichen melancholischen Abschieds gestanden, einer im trübsten Sinne des Wortes ,realistischen“ Desillusionierung, auch wenn sie sich zunächst noch als jugendlichen Aufbruch drapierte. Nach dem Scheitern der bürgerlichen Revolution hatte der junge Theodor Fontane 1853 den Beginn einer neuen, einer programmatisch realistischen Literatur proklamiert. Realistisch, das sollte nun heißen: frei von den romantischen Restbeständen, als die das utopische Potential der jungdeutschen und Vormärzliteratur nun noch

9 „Riechen wir noch Nichts von der göttlichen Verwesung? - auch Götter verwesen! Gott ist todt! Gott bleibt todt! Und wir haben ihn getödtet! Wie trösten wir uns, die Mörder aller Mörder?" (Friedrich Nietzsche: Die fröhliche Wissenschaft, Abschnitt 125: Der tolle Mensch. In: Ders.: Sämtliche Werke. Kritische Studienausgabe. Hg. von Giorgio Colli und Mazzino Montinari, München, 2. Aufl. 1988, Bd. 3, S. 480f., hier S. 481)

10 „Der Wille bewegt nichts mehr, erklärt folglich auch nichts mehr - er begleitet bloss Vorgänge, er kann auch fehlen. Das sogenannte ,Motiv‘: ein andrer Irrthum. Bloss ein Oberflächenphänomen des Bewusstseins, ein Nebenher der That, das eher noch die antecedentia einer That verdeckt, als dass es sie darstellt. Und gar das Ich! Das ist zur Fabel geworden, zur Fiktion, zum Wortspiel: das hat ganz und gar aufgehört, zu denken, zu fühlen und zu wollen!“ (Friedrich Nietzsche: Götzen-Dämmerung, Kapitel: Die vier grossen Irrthümer, Abschnitt 3. In: Ders.: Sämtliche Werke. Kritische Studienausgabe. Hg. von Giorgio Colli und Mazzino Montinari, München, 2.Aufl. 1988, Bd. 6, S. 91)

11, ,...] daß das Ich nicht Herr [ist] in seinem eigenen Haus“ (Sigmund Freud: Eine Schwierigkeit der Psychoanalyse [1917]. In: Ders.: Gesammelte Werke, Bd. 12, London 1947, S. 11). 
wahrgenommen wurde. Bereits in diesen Anfängen, in denen im Programm der literarischen Bewegung - die um diese Zeit noch mehr Programm ist als Poesie noch viel Hegel'sche Geschichtsphilosophie herumspukt, inmitten des unternehmungslustigen Optimismus lauert die Furcht vor einer womöglich absurden Zielund Sinnlosigkeit des Daseins, der Welt, der Geschichte, die nur ,poetisch“ auf Distanz gebracht, anschaubar gemacht und kompensiert werden könnte - einer Welterfahrung, die sich in den rekurrenten Bildern von Sterben und Tod nur am drastischsten artikuliert. Hinter Hegel wartet vom Beginn des Poetischen Realismus an bereits Schopenhauer. Das „Ende der Kunstperiode“ hatten Hegel und Heine noch in der Absicht ausgerufen, einem neuen, optimistischen Geschichtsverständnis und womöglich gar der revolutionären Tat freie Bahn zu schaffen. ${ }^{12}$ Aber dem Ende der Kunstperiode folgte der „Tod Gottes“, der Tod des Subjekts, das Ende der verbindlichen Gewissheiten.

II.

Kein Erzähler des Poetischen Realismus hat diesen wahrhaft universalen Pessimismus des Zeitgeistes, seine heimliche Todesnähe so scharf wahrgenommen, so präzise protokolliert und so konsequent in Erzählverfahren umgesetzt wie Wilhelm Raabe. Keiner unter den bürgerlichen Realisten hat zugleich wie er - und schon dieses Zugleich bezeichnet eine seiner großen Leistungen - die konkreten, in explodierenden Metropolen wie Berlin unübersehbaren sozialen Verwerfungen als ein Menetekel wahrgenommen, das den Untergang dieser so verblendet selbstgewissen Bürgerkultur ankündigte; keiner hat wie er, der Bewunderer und

12 Die Formulierung vom „Ende der Kunstperiode“ gebraucht Heine, in unterschiedlichen Varianten, seit seiner Rezension über Wolfgang Menzels „Die Deutsche Literatur“ 1828: „Ist doch die Idee der Kunst zugleich der Mittelpunkt jener ganzen Literaturperiode, die mit dem Erscheinen Goethes anfängt und erst jetzt ihr Ende erreicht hat" (Heinrich Heine: Historisch-kritische Gesamtausgabe der Werke, Bd. 10, Hamburg 1993, S. 239). Die wohl breiteste Wirkung erlangte die Wendung von der „Endschaft der ,goetheschen Kunstperiode', mit welchem Namen ich diese Periode zuerst bezeichnete“, zu Beginn der „Romantischen Schule“ 1835 (Heinrich Heine: Die romantische Schule. Erstes Buch. In: Ders.: Historisch-kritische Gesamtausgabe der Werke, Bd. 8/1, Hamburg 1979, S. 125; zum Kontext vgl. Jeffrey L. Sammons: Heinrich Heine, Stuttgart 1991). Tatsächlich lassen sich sehr ähnliche Formulierungen schon früher bei Friedrich Schlegel nachweisen (dazu Ernst Behler: Die Wirkung Goethes und Schillers auf die Brüder Schlegel. In: Unser Commercium. Goethes und Schillers Literaturpolitik. Hg. von Wilfried Barner, Eberhard Lämmert und Norbert Oellers, Stuttgart 1984 [= Veröffentlichungen der Deutschen Schillergesellschaft, Bd. 42], S. 559-583, hier S. 560, sowie Günter Häntzschel: Das Ende der Kunstperiode? Heinrich Heine und Goethe. In: Goethes Kritiker. Hg. von Karl Eibl und Bernd Scheffer, Paderborn 2001, S. 57-70). Zum Zusammenhang mit Hegels Wendung vom „Ende der Kunst“ in den „Vorlesungen über Ästhetik“ Michael Baumgarten und Wilfried Schultz: Topoi Hegelscher Philosophie der Kunst in Heines „Romantischer Schule“. In: Heine-Jahrbuch 17 (1978), S. 55-94, hier S. 79. 
Nachfolger von Charles Dickens auch hier, die tödlichen Auswirkungen einer rücksichtslosen Industrialisierung so konsequent als Symptome eines Geschehens erkannt, das im Zeitalter des Imperialismus mit ungeheurer Beschleunigung globale Ausmaße annahm - so dass Pfisters Mühle, dieser mit Recht vielgerühmte erste ,ökologische Roman' der deutschen Literatur, aus den hyperrealistischen Schilderungen eines lokalen Gewässersterbens einigermaßen rasant die Vision einer globalen Zerstörung ableitet. Zwar wird sie vorsichtshalber einer nicht sehr vertrauenswürdigen Romanfigur in den Mund gelegt, dem trunksüchtigen Dichter Lippoldes, der diese Vision unterm Tannenbaum des bürgerlichen Weihnachtsfestes herauskreischt. Aber höchst subtil werden seine Untergangsvisionen vom Erzähler beglaubigt, in diskreten und leicht zu überlesenden Anspielungen, und die höhnisch-satirische Schärfe des lyrisch-epischen Generalangriffs ist erst im Expressionismus wieder erreicht worden. ${ }^{13}$

Kein Erzähler des Poetischen Realismus hat schließlich das Ende aller geschichtsphilosophischen Sinngebungsversuche so radikal formuliert, wie Raabe es bereits in seinen frühen Weltanschauungsgedichten proklamiert und dann in seinen anti-historistischen Geschichtsromanen und -erzählungen realisiert hat. 1859 hat der junge Schopenhauerianer die Verse geschrieben:

Im Jammer kämpft die Menschheit sich ins Grab

Durch Tag und Nacht; o schales Einerlei,

Das Flut auf Flut rollt in das Nichts hinab,

Und nimmer ist das Puppenspiel vorbei! [BA 20, S. 354]

Im Jahr 1861 hat er dem die Verse folgen lassen:

Licht und Erde Meer und Seelen

Löschet die Vernichtung aus [...]. [BA 20, S. 386]

Raabe gestaltet diese dunkle Seite seines - und vielleicht nicht nur seines - Jahrhunderts in einer, wie mir scheint, beispiellosen Konsequenz sein Lebenswerk hindurch, buchstäblich vom ersten Satz seines ersten Buches an (das in der künftigen Reichshauptstadt spielt, aber nur von der „Sperlingsgasse“ erzählt wie von einer widerständigen kleinen Gegenwelt inmitten der großen): „Es ist eigentlich eine böse Zeit! [...] Auf der Ferne liegen blutig dunkel die Donnerwolken des

13 Zu dem im Folgenden skizzierten Zusammenhang ausführlicher mein Aufsatz: Ökologische Krise und ästhetische Innovation im Werk Wilhelm Raabes. In: JbRG 1992, S. 1-27; grundlegend Dirk Göttsche: Zeitreflexion und Zeitkritik im Werk Wilhelm Raabes, Würzburg 2000, S. 99-109; Harro Segeberg: Literatur im technischen Zeitalter. Von der Frühzeit der deutschen Aufklärung bis zum Beginn des Ersten Weltkrieges, Darmstadt 1997, S. 183-189; Markus Winkler: Die Ästhetik des Nützlichen in „Pfisters Mühle“. Problemgeschichtliche Überlegungen zu Wilhelm Raabes Erzählung. In: JbRG 1997, S. 18-39; Axel Goodbody: From Raabe to Amery: German Literature in Ecocritical Perspective. In: From Classical Shades to Vickers Victorious. Hg. von Steve Giles und Peter Graves, Bern [u.a.] 1999, S. 77-96. 
Krieges, und über die Nähe haben Krankheit, Hunger und Not ihren unheimlichen Schleier gelegt“" (BA 1, S. 11). Und er erzählt davon bis zum letzten Buch, dem vollendeten Fragment Altershausen (abgebrochen 1902), dessen greiser Held um die Wende vom 19. zum 20. Jahrhundert einen Spaziergang auf den längst geschleiften Wällen der Stadt Braunschweig unternimmt und bemerken muss, dass in der industrialisierten Großstadt die Schmetterlinge als „Ungeziefer“ verschwunden sind, Vorboten eines ökologischen Untergangs: „daß sich ihm hier, grade hier, [...] die kalte Teufelsfaust entgegenballen würde, hatte er sich auch nicht vermutet. Es war aber so.“ (BA 20, S. 212) ${ }^{14}$

Diesen literarischen Zeugnissen entspricht eine zunehmende Todes-Obsession des Autors selbst, die sich, wo sie sich philosophisch selbst reflektiert, gewiss auf Schopenhauer'sche Begriffe zurückführen lässt, ebenso gewiss aber in ihnen allein nicht mehr aufgeht. 1870 schreibt seine Frau Bertha an Marie Jensen, der Wahlspruch ihres Ehemannes laute eigentlich: „Ich werde nur in meiner Todesstunde glücklich sein!“ (BA Erg.-Bd. 3, S. 116) Das Wort Solons und des Buches Jesus Sirach, dass niemand vor seinem Tode glücklich zu preisen sei, nimmt da die Formen des Sophokles-Wortes an, nie geboren zu sein sei das Beste, das Zweitbeste aber, bald zu sterben. ${ }^{15}$ In Raabes Notizen heißt das zehn Jahre später: „Das Beste, was der Mensch im Leben haben kann, ist ein Stück von dem, was er im Tode ganz haben wird - Ruhe."16 Der alte Raabe, so erinnert sich seine Tochter später, habe zu seinen Kindern geradezu redensartlich den Satz gesagt: „Wartet nur, wenn der große Weltkrieg kommt“. ${ }^{17}$ Immer wieder, bis zu seinem Tod im Jahr 1910, vor hundert Jahren.

Aber was heißt „Sterben“ in diesem unübersehbar weitläufigen Lebenswerk, wie ist die epochale Leitmetapher in ihm literarisch ausbuchstabiert? Vor allem, so scheint mir, in der kunstvollen Überlagerung unterschiedlicher Dimensionen. Das reale individuelle Sterben kann kollektive, ja globale Sterbevorgänge und damit Epochenzusammenhänge repräsentieren - metonymisch als ein Teilereignis, etwa als Tod eines Soldaten in einem Krieg, oder metaphorisch als Sinnbild eines andersartigen, aber auf bestimmte Weise ebenfalls vom Tod bestimmten Zusammenhangs. Das sehen wir uns genauer an.

Das individuelle Sterben wird in Raabes Erzählungen umfangreich und ostinat zum Thema. Wie auch immer zeichenhaft überhöht, symbolisch oder allegorisch

14 Zur Verknüpfung von kollektiven und individuellen Verlusterfahrungen und Todesängsten und ihrer narrativen Bewältigung in diesem Roman jetzt Torsten Vo $\beta$ : Narrative des Alters. Wilhelm Raabes „Altershausen“. Erzählerische Kompensationsstrategien des Zeitund Präsenzverlustes. In: Signaturen realistischen Erzählens im Werk Wilhelm Raabes. Anlässlich des 100. Todestages hg. von Dirk Göttsche und Ulf-Michael Schneider, Würzburg 2010, S. 214-230.

15 Sophokles: Ödipus auf Kolonos, 5. Akt, Vers 1225.

16 Raabe: Einfälle (wie Anm. 1), S. 193, datiert auf den 26. Oktober 1880.

17 So Klara Behrens-Raabe 1931: „Wir [...] sind aufgewachsen mit der ernsten Warnung: ,Wartet nur, wenn der große Weltkrieg kommt [...].“ (BA Erg.-Bd. 4, S. 79) 
repräsentativ die Schilderungen individuellen Sterbens erscheinen - es ist in Raabes Texten doch zunächst immer das sehr genau beobachtete, eindringlich vergegenwärtigte Sterben eines jeweils einzelnen Menschen. Ich wüsste keinen anderen Erzähler des Poetischen Realismus zu nennen, in dessen Werk so viel und so ausführlich gestorben wird (in der Lyrik wäre sein großer Antipode Theodor Storm zu nennen, der seine Dichtung ja ausdrücklich, und aus ähnlichen Ursachen wie Raabe sein Erzählwerk, unter das Signum „Leben im Zeichen des Todes“ gestellt hat; ${ }^{18}$ aus der niedersächsischen Nachbarschaft müsste unbedingt auch Wilhelm Busch einbezogen werden, der Verfasser der Bildergeschichten wie der unterschätzte und abgründige Autobiograph). ${ }^{19}$

Raabes fiktive Lebensläufe sind Sterbensläufe - vom alten Mann eben, der im Debütroman eines gerade sechsundzwanzigjährigen Autors mit großer Abschiedsgeste auf das Leben zurückblickt, über den durch die Weltgeschichte rumpelnden Totenkarren des Schüdderump bis zu Unruhige Gäste, Im Siegeskranze, Im alten Eisen, Das Odfeld, Die Akten des Vogelsangs, Altershausen - und das in ebenjener Epoche, über die der Historiker Philippe Ariès in seiner Geschichte des Todes geschrieben hat, dass in ihr das Sterben so privatisiert und tabuisiert worden sei wie nie zuvor. ${ }^{20}$

Erst wenn dies festgehalten ist, darf man sagen, dass das Sterben eines einzelnen Menschen bei Raabe fast immer zugleich ein epochales Geschehen repräsentiert; das eine spiegelt sich im anderen. Die poetische Kunst und ineins damit die moralische Qualität dieser Darstellungen liegt dabei, scheint mir, vor allem in der vollkommenen Balance des Epochalen und des Individuellen. Wie leicht wäre es möglich gewesen, wie nahe hätte es gelegen, die individuellen Sterbefälle zu verkürzen, die Sterbenden selbst zu erniedrigen zu Allegorien historischer Prozesse, die sich in ihnen verkörpern und als Personen anschaubar werden! Und wie leicht hätten sich umgekehrt diese Personen zu Objekten einer Rührung vergrößern lassen, deren sentimentale Gefühlsintensität den kühlen Blick auf die epochalen Zusammenhänge und Bedingungen ihres Sterbens verstellt, die historische und politische Trauerarbeit in bloßer Rührung sich hätte ausweinen lassen! Wie vollkommen aber erscheinen beide Perspektiven im Gleichgewicht, wie ausgewogen sieht man Individualität und Epochengeschehen in ein Verhältnis wechselseitiger

18 So die berühmte Gedichtüberschrift in Theodor Storm: Gedichte, Novellen 1848-1867. Hg. von Dieter Lohmeier, Frankfurt/Main 1987 (= Sämtliche Werke in vier Bänden, Bd. 1; Bibliothek deutscher Klassiker, Bd. 19), S. 62.

19 Dazu grundlegend Gert Ueding: Wilhelm Busch. Das 19. Jahrhundert en miniature. Erweiterte und revidierte Neuausgabe, Frankfurt/Main und Leipzig 2007.

20 Philippe Ariès: Geschichte des Todes. München und Wien 1980 (u.ö.; französische Erstausgabe u.d.T. „L'Homme devant la mort“, Paris 1977). - Mit den genannten Romanen und Erzählungen habe ich mich ausführlich in einer Studie beschäftigt, auf deren Erträge ich mich im Folgenden mehrfach beziehe, ohne das jeweils im Einzelnen nachzuweisen: Theodizee und Erzählverfahren. Narrative Experimente mit religiösen Modellen im Werk Wilhelm Raabes, Göttingen 1990 (= Palaestra, Bd. 289). 
Erhellung treten, wenn man die Sterbeszene der armen Ludowike in der Novelle Im Siegeskranze noch einmal nachliest - oder den Tod des jugendlichen Draufgängers Thedel von Münchhausen, der als exemplarisches Opfer nicht nur des Siebenjährigen, sondern des von Raabe gesehenen heimischen und kolonialen Weltkriegs exemplarisch sinnlos fällt.

Die nationale Erfolgsgeschichte Deutschlands von den Befreiungs- zu den Vereinigungskriegen - in Raabes Novellen und Romanen sind es stets die Gescheiterten, die Außenseiter und Verlierer, aus deren Perspektive sie umerzählt wird. In Im Siegeskranze ist es eine alte Frau, die ihrer Enkelin die Ereignisse von 1812 und 1813 als die Geschichte der durch keine nationale Sinnbehauptung zu tröstenden Leidensgeschichte der Opfer berichtet. Denn die Heldin, die am Ende wahrhaftig „Im Siegeskranze“ dasteht wie in der Glorie einer heiligen Märtyrerin: sie ist ja nur eine der Hinterbliebenen der siegreichen Soldaten, und aus Verzweiflung ist sie wahnsinnig geworden. Wie ihre Schwester um sie trauert, so der greise Noah Buchius auf dem Odfeld um seinen Lieblingsschüler Thedel von Münchhausen, und seine Frage danach, warum der junge Mann gefallen sei, der alte aber überlebt habe, findet keine Antwort in dieser Nacht und diesem Nebel, in denen sich der Gang der Weltgeschichte als unendlicher Kreislauf erweist, als „schales Einerlei“ und makabres „Puppenspiel“", als eine ewige Wiederkunft der immer gleichen Vernichtung (BA 17, S. 354, 386). Dieser Nebel lichtet sich nicht, diese Nacht endet nie. Oder man denke an jene großartige und aus unserer Rückschau besonders deutlich über ihre Zeit hinausweisende Novelle, die Raabe gegen den zeitgenössischen und jeden Antisemitismus geschrieben hat, Höxter und Corvey. ${ }^{21}$ Hier flackert unvermittelt die Möglichkeit eines Massenmordes auf; der Verfasser des ,Jugendquarks“ vom Hungerpastor ahnt sehr wohl, welche Möglichkeiten mit dem Schlachtruf „Nun auf die Juden!“22 inmitten der trügerischen christlichen und bürgerlichen Normalität aufbrechen. In dieser WeserNovelle, in der das Juden-Pogrom in der provinziellen Kleinstadt nur ein beinahe anekdotisches Ereignis am Rande eines unablässig gewalttätigen Weltgeschehens zu sein scheint, hier steht dann auch der schaurige Satz, der die präzise beobachteten besonderen Vorgänge dieser einen Nacht ins Exemplarische erhebt: „Wenn einer in dieser Nacht in Höxter an der Weser Vernunft gesprochen hatte, so war das der Tod gewesen." 23

Überall dort, wo das individuelle Sterben Raabe'scher Roman- und Novellenfiguren so eng mit epochalen Krisenerfahrungen verbunden ist wie im Odfeld

21 Dazu - und gegen das hartnäckige Vorurteil von Raabes ,Antisemitismus * grundlegend Hans-Jürgen Schraders Nachwort in seiner mustergültigen Neuausgabe dieser noch immer zu wenig bekannten Toleranznovelle: „Höxter und Corvey“. Tragisches Erleben und humoristischer Freiblick in verworrenen Zeiten. In: Wilhelm Raabe: Höxter und Corvey. Eine Erzählung. Nach der Handschrift von 1873/74 hg. von Hans-Jürgen Schrader, Stuttgart 1981, S. 189-213.

22 Raabe: Höxter und Corvey (wie Anm. 21), S. 64.

23 Ebd., S. 64 und 96. 
oder in den Akten des Vogelsangs - und das gilt ja bei näherem Hinsehen für die allermeisten dieser Sterbensläufe -, überall dort kann seine Darstellung sich unvermittelt öffnen in Visionen eines nicht nur kollektiven, sondern geradezu eines globalen Sterbens. Dieser Blick kann auf die geschichtliche Vergangenheit gerichtet sein, auf die unmittelbare Gegenwart oder auf die Zukunft, und nicht selten auf alle drei Zeiten zugleich - so wenn der Herzog Ferdinand, der vermeintliche Kriegsherr selbst während eben der Schlacht auf dem Odfeld, in der Buchius überlebt und Münchhausen stirbt, die Weltgeschichte als „eine Schlächterei ohne Ende“ erkennt, bestialisch-endlose Wiederkunft des Gleichen (BA 17, S. 175).

Auch dieser Gedanke lag in der Luft. Theodor Storm, von seinem Sohn Ernst um ein patriotisches Wort zum deutsch-französischen Krieg gebeten, antwortet im August 1870 mit den großartigen Sätzen:

Was mich hauptsächlich beherrscht - und das verschlingt alles Andere - das ist der Ekel, einer Gesellschaft von Creaturen anzugehören, die außer den übrigen ihnen von der Natur auferlegten Funktionen des Futtersuchens, der Fortpflanzung etc auch die mit elementarischer Stumpfheit verfolgt, sich von Zeit zu Zeit gegenseitig zu vertilgen. ${ }^{24}$

Es ist derselbe Grundgedanke wie bei Raabe. Aber nur er hat aus ihm auch die äußerste literarische Konsequenz gezogen, indem er seinen Anti-Geschichtsroman inszeniert als eine realistische Wiederkehr der biblischen Apokalypse - einer Apokalypse jedoch ohne Ende.

Am rasantesten vollzieht sich der Übergang vom geradezu kleinmeisterlich geschilderten realistischen Alltagsgeschehen in eine globale Untergangsvision im schon erwähnten Weihnachtskapitel von Pfisters Mühle. Und der visionäre „Kehraus“ im Weltuntergangsgedicht dort ${ }^{25}$ wird uns wiederum als reale, gewissermaßen im bürgerlichen Kleinformat inszenierte Apokalypse in den Akten des Vogelsangs wieder begegnen; auch hier nennt Velten Andres seine finale Abrechnung mit dem Eigentumsdenken seinen „Kehraus“, und die Zuschauer denken ans ,Jüngste Gericht“ (BA 19, S. 379).

In solchen Überlagerungen unterschiedlichster Dimensionen des Sterbens vom individuellen Schicksal über die kollektive Bewegung zum globalen Geschehen, in solchen intertextuellen Schichtungen einander bestreitender Sinnangebote, in solchen gleitenden Übergängen vom sehr konkret erlebten physischen Sterben zu Sterbens-Figurationen als Metaphern einer transzendentalen Obdachlosigkeit - in alldem wird in Raabes Texten zu Ende gedacht und erzählend zu Ende gebracht, erledigt, was dem gebildeten bürgerlichen Jahrhundert an Weltdeutungsmodellen lieb und teuer war.

24 Theodor Storm / Ernst Storm: Briefwechsel. Kritische Ausgabe. Hg. von David L. Jackson, Berlin 2007, S. 73 (Brief Nr. 35, 3. August 1870).

25 ,Sehr drollig wird das sein für den, der da zuletzt lacht, / Sieht er im Wirbel fliegen, was ihn quälte, / Bis selber ihn der letzte Kehraus faßt.“ (BA 16, S. 86) 
Unbeirrbar - oder auch: unbelehrbar - misstraut Raabe deshalb in lebenslang wachsender Skepsis allen geschichtsphilosophisch begründeten Lösungsangeboten, die er, durch Schopenhauer frühzeitig desillusioniert, als ersatzreligiöse Systeme begreift und mit dem religiösen Dogma verwirft. In der Folge seiner frühen Religionskritik bleibt im späteren Werk keine der kurrenten Ersatzreligionen ungeschoren, weder die künstlerischen (dem Werben der Wagnerianer widersteht er wacker) noch die philosophischen (den Nietzsche-Kult sieht er mit Argwohn) noch gar die politischen. Je älter er wird, desto argwöhnischer zeigt er sich gegenüber den hochfliegenden, zeitweise geradezu utopischen Hoffnungen auf eine freie bürgerliche Republik, an deren Zukunft er nicht mehr glauben kann, seit - wie er in Altershausen melancholisch und sarkastisch formuliert - das „flüchtig[e] Niedersteigen des Reichs der Himmel auf die Erde“ (BA 20, S. 257) in der Revolution von 1848 einem bewaffneten Nationalismus und Kolonialismus gewichen ist. In derselben Weise, mit demselben Bild formuliert er sein Misstrauen gegenüber dem sozialistischen „Zukunftsstaat“, mit dem ausgerechnet er, der scharfe Sozialkritiker, doch nichts zu tun haben will. „Er weiß nämlich“, spottet er im Alten Eisen über einen fortschrittlichen Intellektuellen, „wenn er in Stimmung ist, über alles göttlich zu reden, und wenn es ihm aus Zufall zwischen die Lippen kommt, auch himmlisch sozialdemokratisch“" (BA 16, S. 476). Weil er nicht mehr an den Himmel glaubt, deshalb misstraut er nun allen, die ihm einen Himmel auf Erden zu versprechen scheinen - wie er seiner bewundernden und selbst bewundernswerten Leserin Clara Zetkin in fast verletzender Schärfe mitteilt, ${ }^{26}$ der großen Frauen- und Bürgerrechtlerin, die viel später Mitglied im Zentralkomitee der Kommunistischen Partei wurde und isoliert im Exil in Stalins Sowjetunion starb.

Es wäre Raabe - dem großen Schopenhauerianer, als den Søren Fauth ihn uns so überzeugend vor Augen geführt hat ${ }^{27}$ - vermutlich nicht recht gewesen, das zu hören, aber je länger, desto deutlicher liest er sich wie der feindliche Bruder des anderen großen Schopenhauerianers. Je länger, desto mehr wird Raabe zu einer Art Anti-Nietzsche, im doppelten Sinne des Anti: in Analogie und Opposition. Ich meine damit jetzt nicht weitere intertextuelle Beziehungen (so produktiv gerade solche Spurensuchen in der jüngeren Raabe-Forschung immer wieder gewesen sind). Es ist für mein Thema durchaus zweitrangig, inwieweit hier direkte Genealogien vorliegen oder nur Analogien. Worauf es mir ankommt ist zu zeigen, dass Raabes Werk dort, wo es der Diagnose gilt, die das „Sterben“ zur bloß verleugneten Epochensignatur erhebt, streckenweise auf durchaus ähn-

26 Brief an Clara Zetkin vom 10. März 1908 (BA Erg.-Bd. 2, S. 477: Brief 462).

27 Søren R. Fauth: Der metaphysische Realist. Zur Schopenhauer-Rezeption in Wilhelm Raabes Spätwerk, Göttingen 2007; ders.: Die gegenseitige Mörderei und die geniale Anschauung. Raabes „Odfeld“, „Stopfkuchen“ und die Philosophie Schopenhauers. In: „Die besten Bissen vom Kuchen“. Wilhelm Raabes Erzählwerk: Kontexte, Subtexte, Anschlüsse. Hg. von Søren R. Fauth, Rolf Parr und Eberhard Rohse, Göttingen 2009, S. 135-166. 
lichen Denkwegen verläuft wie in Nietzsches radikalen, in der Proklamation des „Todes Gottes“ kulminierenden Zeitdiagnosen.

Am komprimiertesten zeigt sich das, was ich meine, in Raabes Roman Im alten Eisen. $\mathrm{Ob}$ als bewusste Kontrafaktur oder in unabhängiger Bezugnahme auf dieselben mythischen Bilder - hier sind das Dionysische und die beim Wort genommene Jesusnachfolge, Dionysos und der Gekreuzigte in engster dialektischer Verschränkung aufeinander bezogen, in einem ganz und gar der Humanität verpflichteten, darum zugleich mitleidvollen und humoristischen Buch, geschrieben über eine eigentlich alptraumhafte Welt „im Sterben“. Denn Raabe, der dezidierte Nicht-Sozialist, schildert auch den Krieg der Klassen mit bemerkenswerter Konsequenz aus der Perspektive der Opfer, in ganz ähnlicher Weise wie die Innenansichten der militärischen Kriege - und wie diese in einer virtuosen Überblendung des Individuellen mit dem epochal Allgemeinen. Die suggestive Schilderung der proletarischen Stadtviertel in Im alten Eisen inszeniert die Kreuzberger Mietskaserne als Totenwelt. In dieser Hölle scheint der „Tod Gottes“ ebenso vorausgesetzt wie auf dem Schlachtfeld der Weser-Trilogie. Wie dort die Apokalypse im Gegensatz zu den biblischen Prätexten keinen ,neuen Himmel', keine ,neue Erde' hervorbringt, sondern sich nur endlos fortsetzt - so inszenieren die gehäuften Dante-Anspielungen hier die Großstadtwelt als ein unaufhörliches Inferno. Ausgerechnet dem einzigen Bildungsbürger, den es als eine Art Stellvertreter des erschrockenen Lesers - in diese Abgründe verschlagen hat, ausgerechnet diesem Kunstliebhaber und Literaturkenner bleibt es überlassen, diese Identifikation auszusprechen. ${ }^{28}$ Eigentlich nur als gebildete Entlastungsphantasie soll ihm ersichtlich der Vergleich dieses übervölkerten und verelendeten Mietshauskomplexes mit Dante dienen - und gleichsam über seinen Kopf hinweg begreifen die Leser (und etwas später begreift er es auch selbst), dass dies ganz buchstäblich die Hölle ist.

Dass hier zwei Proletarierkinder, die letzten, ohnmächtigen Glieder in der hierarchischen Folge der sozialen Deklassierungen, hilflos am Leichnam der toten Mutter verharren mussten, ist da nur eine Episode unter anderen; dass wenn nicht die Heldin, so doch jedenfalls die zentrale Gestalt dieses Romans die Leiche selber ist, die tote Mutter - das ist der radikalste Ausdruck einer heillosen Ungeborgenheit, eines Ausgesetztseins, aus dem die Ausgesetzten keinen Weg zurück in die Welt des Lebens finden können. Von hier aus geht es geradewegs auf den Friedhof; und der umständliche Weg dorthin beschreibt eigentlich auch schon die ganze Romanhandlung - beinahe jedenfalls. Denn dass es überhaupt zum Friedhof geht und von dort am Ende doch wieder zurück in die Welt der Lebenden, das liegt am ganz nüchternen, pragmatisch-tätigen Eingreifen der bunt zusam-

28 BA 16, S. 441f. Raabe zitiert nach der Übersetzung seines Freundes Friedrich Notter: Dante Alighieri's Göttliche Komödie, übersetzt und erläutert von Friedrich Notter. Bd. 1: Die Hölle, Stuttgart [1871]. 
mengewürfelten Gemeinschaft aus dem „Alten Eisen“, einer Gemeinschaft, die erst aus diesem Anlass und zu diesem Zweck zur Gemeinschaft wird. ${ }^{29}$

Deren Initiator ist der zur realistischen Figur eines Wanderarbeiters mutierte Schmied von Jüterbog; er heißt Peter Uhusen. Und dieser vitale Helfer ist nun eine derart dionysische Figur, dass ich bei anderer Gelegenheit Raabe eine zumindest oberflächliche Kenntnisnahme von Nietzsches Tragödienschrift unterstellt habe. Uhusens stehendes Accessoire ist der ,Spazierstock [...] mit einer Bocksfratze am Griff“, der den Roman hindurch leitmotivisch als „Faunenfratze“ und „Satyrfratze“ variiert wird. ${ }^{30}$ Als neuer Dionysos wird Uhusen zum genauen Gegenbild des apollinisches Salonliteraten Brokenkorb - der an seiner Seite wohl oder übel seinen ästhetischen Olymp verlassen und in die „Schrecken und Entsetzlichkeiten des Daseins“ hinuntersteigen muss, als die Nietzsche dort ja die Abgründe unter dem ,olympischen Zauberberg“ beschreibt ${ }^{31}$ - und die hier als Kreuzberger Elendsquartier sehr handfeste Gestalt gewonnen haben. Zugleich aber ist dieser Uhusen ein Nachfolger des Gekreuzigten - ist nach seinen eigenen, ausführlich räsonnierenden Worten auf das geraten, was er die „Wege des Menschensohns“" nennt (BA 16, S. 433). Diese ganz und gar unorthodoxe JesusNachfolge ereignet sich in einer Welt nach dem „Tode Gottes“. So wird dieser resolut diesseitige Jesus-Dionysos zum Lebenshelfer in der Todeswelt.

Ganz anders als Nietzsche, ja in genauem Gegensatz zu ihm fragt Raabe also gerade nicht nach den Möglichkeiten einer ästhetisierenden Lebens- und Selbstgestaltung in dieser Welt, einer Verwandlung des eigenen Lebens in ein Kunstwerk; er verspottet sie vielmehr in der Gestalt des egozentrischen Ästheten Brokenkorb. An die Stelle einer zur Ersatzreligion erhobenen Ästhetisierung setzt er die praktische humane Tat, an die Stelle des sich selbst erschaffenden großen Ich das Eintreten in die und für die Gemeinschaft der solidarisch Helfenden. Weil Uhusen gemeinsam mit den anderen Deklassierten den Kindern, diesen Verlierern der kapitalistischen Nahrungskette, zur Hilfe kommen will, aus keiner anderen Motivation als lebendigem Mitgefühl, weil er von außen eindringt in die Totenkammer - deshalb kann er in einem einfachen und durchaus unsentimentalen Vorgang das Wunder bewirken, dass in diese Höllenwelt auf einmal ein Himmelslicht fällt. Wie deren symbolischer Stilisierung ein religiöses Modell zugrunde lag, wie also das Berliner Arbeiterquartier zum Dante'schen Inferno wurde, so gewinnt nun auch diese solidarische Hilfeleistung eine religiöse Dignität, die freilich ganz in ihrer reinen Menschlichkeit besteht. Dass die Todverfalle-

29 Vgl. Jeffrey L. Sammons: Wilhelm Raabe. The Fiction of the Alternative Community, Princeton/NJ 1987.

30 BA 16, S. 354, 496 und 433. Ausführlicher zu dieser möglich Transformation von Motiven Nietzsches mein Beitrag: Die Geburt der Tragödie im ,alten Eisen“? Vermutungen über Raabes Nietzsche-Lektüre. In: Produktivität des Gegensätzlichen. Festschrift für Horst Denkler zum 65. Geburtstag. Hg. von Julia Bertschik u.a., Tübingen 2000, S. 19-30.

31 Friedrich Nietzsche: Die Geburt der Tragödie. In: Ders.: Sämtliche Werke (wie Anm. 10), Bd. 1, S. 35 . 
nen ins Leben zurückfinden, überdies auf dem so buchstäblichen wie sinnfälligen „Kreuzberg“, das ist Raabes moralisch-pragmatische Version der Auferstehung.

Welcher Gegensatz zum keineswegs mehr anrührenden, vielmehr verzweifelten Bericht vom finalen Rückzug jenes Velten Andres, der in einer ganz auf Besitz und Eigentum gegründeten Welt zum rettungslosen Einzelgänger geworden ist, einsam verklettert im Geäst seines Weltenbaums - und der nun, das Gesicht zur Wand, auf einem Bett in der uterinen Höhle seines letzten Quartiers verendet, am Ende seines Lebens regrediert zum verzweifelten Kleinkind! Wie ein gespenstischer Wiedergänger des vertrackt humoristischen Wunnigel in der gleichnamigen Erzählung erscheint er in diesen letzten Szenen, des Weltverweigerers, der ebenfalls mit dem Gesicht zur Wand stirbt und also mit dem Rücken zu seiner Epoche, die uns Lesern eben in seinem Rücken in ihrer ganzen Abscheulichkeit erkennbar wird.

Ähnlich wie in Im alten Eisen entfaltet sich diese zugleich soziale, moralische und religiöse Stilisierung einer Sterbenswelt, in der unerwartete Lebens-Zeichen sichtbar werden, auch in der Doppelerzählung, die sich aus der Novelle Zum wilden Mann und dem explizit darauf zurückverweisenden Roman Unruhige Gäste ergibt. Drastischer noch als im Inferno des Alten Eisen erscheint hier der Teufel selbst mit allen Merkmalen des gründerzeitlichen Kapitalisten und kolonialen Ausbeuters, enthüllen sich die tödlichen Konsequenzen jener (ins kolonial Globalisierte ausgeweiteten) kapitalistischen Erwerbs- und Besitzideologie, der Velten Andres vergebens den finalen Prozess machen will. Aber auch hier behält das Inferno nur im sozusagen ersten Teil, in der ausweglos pessimistischen Novelle, das letzte Wort. Der darauf antwortende Roman (der ja Teile der Szenerie und der Figurenkonstellation fortschreibt) stilisiert die Lebenswelt der Ausgeschlossenen - diesmal im Dorf, nicht in der Metropole, aber mit denselben Konsequenzen - als eine Welt, in der es alles hinauszulaufen scheint auf Sterbefälle, Begräbnisse und Friedhofsordnungen. Und auch hier werden die Leser am Ende Zeugen eines Vorgangs, in dem dank einer überaus einfachen, beinahe unauffälligen Hilfsbereitschaft im Geist selbstloser und pragmatischtüchtiger Nächstenliebe das Wunder einer Auferstehung geschieht. Wie die Helfer um Uhusen den selbstsüchtigen Bildungsbürger Brokenkorb zum Mithelfer machen, so tritt hier dem mondänen Aristokraten Bielow die schlichte, nicht ohne Ironie als „lutherische Nonne“ eingeführte fromme Phöbe gegenüber (BA 16, S. 274), die den am Typhus erkrankten Dorf-Außenseitern selbstverständlich und selbstlos beisteht (und die ihren Namen einer Helferin des Apostels Paulus im Römerbrief verdankt). ${ }^{32}$

Der zwischen Gesellschaftsrolle, erotischem Begehren und Todessehnsucht taumelnde Kavalier wird sich am Ende mit der tödlichen Krankheit infiziert

32 Röm 16, 1f. - Zur thematischen Nähe der Romane „Im alten Eisen“ und „Unruhige Gäste“ vgl. Göttsche (wie Anm. 13), S. 116-127. 
haben und in visionären Fieberträumen sterben. Phöbe hingegen wird überleben, nach allen Erschütterungen wieder unerschütterlich; mit dem distanzierten Blick des Erzählers auf ihre einsame Gestalt im Lichtkreis der nächtlichen Kammer endet der Roman. Dessen Untertitel lautet: „Ein Roman aus dem Säkulum“. „Säkulum“ kann „Jahrhundert“ heißen; gemeint wäre dann ein Zeitroman. Es kann aber auch in (Phöbes) religiösem Sprachgebrauch verstanden werden: als „Zeitlichkeit“, und so wird das Wort tatsächlich mehrfach im Text selbst übersetzt. ${ }^{33}$ Ein „Roman aus der Zeitlichkeit“ - an diesem Untertitel ist aber auch der unauffälligere erste Bestandteil bemerkenswert. Denn mit dem Wort „Roman“ bezeichnet Raabe von allen Geschichten seines Spätwerks nur diese eine. Die Beziehung zwischen Genre und Zeitlichkeit, die durch diesen eigentümlichen Untertitel sehr diskret angedeutet ist, tritt nun im Verlauf der Erzählung immer deutlicher hervor. Wenn nämlich inmitten der aufgeregten Welt der Unruhigen Gäste Phöbe erscheint, dann wechselt das Erzähltempus vom Präteritum ins Präsens, erst mit ihrem Verschwinden kehrt das gewohnte Tempus zurück, das wieder den Raum fiktionalen Erzählens, fiktionaler Zeitlichkeit signalisiert. Indem Raabe das Präsens ausschließlich mit der Gestalt und Weltsicht Phöbes verbindet, indem er es der „Unruhe“ der im Präteritum geschilderten Gesellschaftswelt gegenüberstellt, verwandelt er die Tempora in grammatische Metaphern - Metaphern nämlich für eine dem Tod verfallene Zeitlichkeit und eine dem Tod enthobene Ewigkeit. Wenn am Ende des Romans die fromme Phöbe allein im Licht ihrer Lampe sitzt, hell inmitten der Finsternis, lebendig inmitten des Todes, dann gerät der Roman in ein Präsens, das nicht mehr ins Präteritum zurückfallen kann: „Phöbe [...] sitzt still in dem engen Lichtkreis“, sie ,ist [...] im Frieden und fürchtet sich nicht“ (BA 16, S. 336f.). Diese Sätze beglaubigen das, wovon sie reden, in ihrer grammatischen Gestalt. ${ }^{34}$

Kenner des Raabe'schen Werks werden hier sehr diskret zurückverwiesen auf jenen Siegeskranz, der in der gleichnamigen Novelle ja vom militärischen Requisit zum Zeichen eines in der einfachen menschlichen Liebe realisierten Heils geworden war, im schärfsten Kontrast zum kollektiven Tötungsrausch der nationalen Kriegsbegeisterung. Und wer will, kann hier auch noch an den Tod der alten, von den Leuten als Hexe verschrienen „Wackerhahnschen“ denken, in Raabes letztem vollendetem Roman Hastenbeck von 1899, die ihrem Leben durch ihre letzte, unspektakuläre und eben deshalb so ganz und gar sinnvolle Liebestat für die schutzbedürftigen Liebenden noch einen völlig hinreichenden

33 Etwa BA 16, S. 294 und 311.

34 Bereits BA 16, S. 295f., werden in der Kontrastierung von mondäner Bade-Welt und Phöbe die Erzähltempora entsprechend semantisiert: Phöbes Erscheinen, bei dem ,alles, was uns die Zeit mißt, auf der Erde still“ steht, wird im Präsens, das „Säkulum“ im Erzähltempus Präteritum geschildert. - Eine ausführlichere Analyse dieser Szene in meinem Beitrag: ,der blinde Fleck der Sprache“. Adnoten zu einem Paradoxon. In: Metaphysik und Moderne. Festschrift für Børge Kristiansen. Hg. von Andreas Blödorn und Søren R. Fauth, Wuppertal 2006, S. 75-88. 
und befriedigenden Sinn gegeben hat. Neben dem dionysischen Uhusen und der frommen Phöbe hat auch sie ihren Platz in Raabes Pantheon - oder sollte man sagen Panoptikum? - der guten Lebens-Geister, der sonderbaren Heiligen, der heiligen Sonderlinge.

Denn immer wieder ist es die einfachste, schlichteste nachbarschaftliche Gemeinschaftshilfe, die Raabe geradezu in die Dimension eines ansonsten nicht mehr geglaubten Heils hinein verklärt - verklärt im ganz wörtlichen Sinne. Mindestens dreimal leuchtet in der todverfallenen Welt der Raabe'schen Erzählungen eine veritable Glorie auf. Sie umleuchtet Ludowike im Siegeskranze der seligen Märtyrer. Sie umgibt Phöbe, die im zeitlos-ewigen Präsens in ihrer Stube sitzt, „im Lichtkreis“. Und genau wie sie sehen wir - und nur wir als Leser - den armen Noah Buchius, den Helfer auf dem Schlachtfeld, ,in einer Glorie, von der er selber am wenigsten wußte“ (BA 17, S. 125).

Was im Licht dieser Glorie sichtbar wird, ist das, was man etwas hochtrabend, aber sachlich gewiss nicht unangemessen, ein post-metaphysisches Ethos nennen könnte, das, indem es sich im Horizont von Leben und Sterben, ja Zeitlichkeit und Ewigkeit situiert, auch Züge einer post-religiösen Religiosität gewinnen kann. Wie sie alle in einer Glorie stehen, von der sie selber am wenigsten wissen, so gehen diese Raabe'schen Vorbildfiguren, ob sie es wissen oder nicht, wie der robuste Uhusen „auf den Wegen des Menschensohns“ (BA 16, S. 433). Weil sie sich in einer physischen Todes-Welt vorfinden, an deren metaphysische Erlösung sie nur ausnahmsweise - wie im Fall Phöbes - glauben, deshalb tun sie Wunder und wecken Tote auf, indem sie sich zu den einfachsten, situativspontanen Hilfsleistungen zusammenschließen, mit denen niemand mehr gerechnet hatte und in deren Vollzug sie einander zum Nächsten werden, manchmal zu Stellvertretern des abwesenden Gottes.

Diese Perspektive gemeinschaftlich-nachbarschaftlichen Handelns lässt sich offenkundig weder als individualistisch noch als kollektivistisch auffassen; viel eher weist sie Gemeinsamkeiten mit dem auf, was man in der neueren Soziologie ,Kommunitarismus“ genannt hat. In der folgenreichen Opposition von Gemeinschaft und Gesellschaft, mit der Raabes Zeitgenosse (und Schüler Theodor Storms) Ferdinand Tönnies in seinem gleichnamigen Werk 1887 die moderne Soziologie begründet, steht die in Raabes Romanen vertretene Position derjenigen der „Gemeinschaft“" nahe und bleibt distanziert gegenüber der auf Institutionen und kodifizierte Regelordnungen gegründeten „Gesellschaft“.35 (Auch deshalb erscheint Stopfkuchen, der zum Selbsthelfer gewordene Selfmademan auf seiner Roten Schanze, als eine bei näherem Hinsehen so hoch ambivalente Gestalt.) Aber Raabes Helfer-Gemeinschaften realisieren sich doch auffallend häufig im Wortsinne anarchisch, im spontanen Handeln mit- und füreinander in

35 Zur Rezeptionsgeschichte ausführlich: Hundert Jahre „Gemeinschaft und Gesellschaft“. Ferdinand Tönnies in der internationalen Diskussion. Hg. von Lars Clausen und Carsten Schlüter, Opladen 1991. 
sozialen Zusammenhängen, die keineswegs so ,gewachsen“ sein müssen, wie das bei den Leuten aus dem Walde auf sentimentale Weise der Fall war und wie sie im Vogelsang gerade katastrophal auseinanderbrechen. Vielmehr können sie sich so unverhofft und vorübergehend, so situativ ergeben wie im Alten Eisen oder auf dem Odfeld. Wer nicht in den Schutzraum einer solchen Gemeinschaft gerät - oder wer sich gar selbst von ihr abwendet -, geht so hilflos zugrunde wie der skurrile Einzelgänger Wunnigel oder der Totalverweigerer Velten Andres.

Jedesmal realisiert sich die Solidarität der nachbarschaftlichen HelferGemeinschaften weder in sozialen Programmen noch in ästhetisierender Drapierung, sondern allein in der konkreten Tat. Für sie finden Raabes Erzählungen immer wieder Bilder von archaischer Einfachheit. Eines dieser wiederkehrenden Leitbilder elementarer Zuwendung ist ganz einfach: die Geste nämlich, einem vom Sterben bedrohten oder erschütterten Menschen mit der Hand über die Stirn zu streichen. So in Im Alten Eisen: Die Kinder haben in der Kreuzberger Armenwohnung Tage und Nächte am Leichnam ihrer Mutter verbracht - nun erscheint endlich die erste, nicht mehr erwartete Helferin. So zeigt uns Raabe die zur Hilfe gekommene Wendeline: „neben dem Strohsack niedergekniet, um dem jüngsten Geschöpf die Haare aus der Stirn zu streichen“ (BA 16, S. 478). So auch in Die Akten des Vogelsangs: Karl Krumhardt sitzt, erschüttert von der Nachricht vom einsamen Sterben seines Jugendfreund Velten, am Schreibtisch, und da tritt seine Frau hinzu ,und legte mir ihre kühle Hand auf die Stirn“ (BA 19, S. 215). Das Schreiben selbst kann nur in Gang kommen dank dieser menschlichen Nähe, dieser Kühlung in der Hitze des menschlichen Gefechts. Nicht anders schließlich auf dem weltgeschichtlichen Schlachtfeld des Odfelds. Hier vollzieht, mitten in Lärm und Getümmel, Noah Buchius eben dieselbe (gleichsam franziskanische) Geste an einer Kreatur, die noch ausgelieferter und hilfloser ist als selbst seine Schutzbefohlenen, einem Pferd: „Er machte auch nicht die Menschheit anders, als sie war. Aber dem dampfenden Tier strich er die triefende Mähne: ,Halt aus, Freund [...]! ““ (BA 17, S. 142f.) Nicht die Menschheit ändern - aber über die Stirn streichen. Was sich in diesem Aber zeigt, das ist vielleicht der Kern von Raabes post-metaphysischer Humanität.

In Gesten wie dieser allein ereignet sich das Wunder, in ihnen aber ereignet sich auch das Wunder ganz: der Trost und die Nähe des „Freundes“ im Augenblick des doch unausweichlichen Endes. Was da geschieht, ist ein Leben mitten im Sterben. Es ist eine Humanität ohne Hoffnung, eine Humanität aus Hoffnungslosigkeit.

III.

„Er starb mit sich und der Welt vollkommen in Frieden“, las Franz Kafka 1921 im Raabe-Gedenkbuch: „Als ihm seine treue Lebensgefährtin mit der Hand über die Stirn strich, sagte er noch: ,Das ist schön.““ In seinem Tagebuch lässt er den 
vorgeblichen Frieden mit sich und der Welt beiseite, reduziert die Szene auf die eine, kleine Geste und fügt nur den Vermerk hinzu, dies sei „Besser zu durchdenken“: „Besser zu durchdenken: Raabe im Sterben, als ihm seine Frau über die Stirn strich: ,Das ist schön."“

Das ist eigenartig. Versteht es sich, habe ich einleitend gefragt, nicht von selbst, dass ein Kranker oder gar Sterbender eine solche Geste eben „schön“ findet? Genau bei dieser scheinbaren Selbstverständlichkeit setzt Kafka ein. Er formuliert nicht die Absicht, die Szene bloß,weiter', sondern den Vorsatz, sie besser zu durchdenken. Und es liegt nahe, das auf den unmittelbaren Kontext zu beziehen, in dem Kafka erklärt, er habe „Vieles durchgelitten in Gedanken“: Im Durchdenken sucht er eine Antwort auf das Durchleiden.

Was Kafka als einziges Detail aus seiner Quelle aufgreift, anstelle aller pathetischen und tiefsinnigen Darstellungen, an denen sie nicht arm ist, das ist diese ganz kleine liebevolle Geste gegenüber dem Sterbenden, der nichts mehr zu gewinnen hat, dieser Trostgeste nach dem Ende der Hoffnung. Nicht diese Geste selbst aber ist es, die er besser durchdenken will, sondern die Antwort des Sterbenden: „Das ist schön“. Denn in dieser existenziellen Grenzsituation wird die Redensart entautomatisiert, hier ist sie mit einem Schlag wieder beim Wort zu nehmen - und zwar als ein nicht mehr ästhetisches, sondern nun als ein ethisches Diktum. Zu betonen wäre der Satz dann nicht mehr umgangssprachlich ,das ist schön', sondern gegen den Strich: ,das ist schön“.

Eigentlich brauchen wir gar nicht über den Sinn dieser Notiz zu spekulieren. Denn erfreulicherweise hat Kafka selbst sie an anderer Stelle interpretiert, in einer kurzen und zu Lebzeiten unveröffentlichten Erzählung, die sich offensichtlich aus ebendieser Raabe-Notiz heraus entwickelt hat. Auf diesen Entstehungszusammenhang hat bereits Hartmut Binder 1975 beiläufig hingewiesen. ${ }^{36}$ Ich meine die wenig bekannte Geschichte Das Ehepaar, die Kafka einige Monate nach seinem Tagebucheintrag in seinem Notizbuch entworfen und dann als ein „offensichtlich [...] abgeschlossenes [...] Werk" in Reinschrift übertragen hat. ${ }^{37}$ Sie erzählt von einem alten Mann, der im Sterben liegt. In der Handschrift heißt er ,K.'; in Max Brods Druckfassung erscheint er durchweg irrtümlich als ,N. Durch die liebevolle Berührung seiner Ehefrau - sie küsst die Hand des, wie sie glaubt, nur Schlafenden - wird der Sterbende wunderbarerweise wieder zum Leben erweckt. Der Ich-Erzähler, ein Handelsreisender, wird zufällig Zeuge des Vorfalls. Da er vom unwiderruflichen Tod des Alten überzeugt war, fühlt er sich angesichts der Szene betroffen an seine eigene, frühverstorbene Mutter erinnert -

36 Hartmut Binder: Kafka-Kommentar zu sämtlichen Erzählungen, München 1975, S. 297.

37 Manfred Engel: Das Ehepaar. In: Kafka-Handbuch. Leben - Werk - Wirkung. Hg. von Manfred Engel und Bernd Auerochs, Stuttgart und Weimar 2010, S. 360f., hier S. 360. Der Text findet sich in Franz Kafka: Nachgelassene Schriften und Fragmente II. Hg. von Jost Schillemeit, Frankfurt/Main 1992, S. 516-524. 
denn, so denkt er, „,die konnte Wunder tun““.38 Beschämt von der Unschuld der Frau und empört über die Kälte eines anderen Besuchers verlässt er das Haus; und damit endet der sonderbare kleine Text.

Er verdankt offenbar noch weitere Details als allein die ,besser zu durchdenken[de]“ Trost-Szene dem Raabe-Gedenkbuch, vom unerwarteten Einschlafen bis zum Verfall des vormals hochgewachsenen Greises. Vor allem aber richtet er seine Aufmerksamkeit auf die Rolle der Helferin. Wie Kafkas K. dem sterbenden Dichter, so ähnelt seine Ehefrau derjenigen Bertha Raabe, an die im Raabe-Gedenkbuch Paul Wasserfalls Beitrag über Wilhelm Raabes Letzte Krankheit und Sterben erinnert. Ihre selbstlose, ja selbstvergessene Hingabe steht im Mittelpunkt seines Berichts: Vorbild für das „unendliche Vertrauen“ dieser „Unschuldigen“, ${ }^{39}$ mit dem die Ehefrau in Kafkas Erzählung den anscheinend Toten wie selbstverständlich wieder zum Leben erweckt wie einen zweiten Lazarus - in einer Glorie, von der sie selber am wenigsten weiß. Das ist schön.

Sonderbare Heilige und heilige Sonderlinge habe ich Raabes Ludowike und Uhusen und Phöbe und Buchius genannt, diese von sich selbst nichts wissenden, an sich selbst nicht denkenden Helden des Lebens in einer Welt des Sterbens. Kafkas unschuldig-arglose, in ihrer Liebe wundertätige Frau K. steht in dieser Reihe. Man kann davon etwas in Raabes Sterbebericht wiederentdecken - vorausgesetzt, man liest und durchdenkt ihn gewissermaßen mit Raabe'schen Augen. Das, scheint mir, hat Franz Kafka getan. Und darum lohnt es vielleicht, an Raabes Todestag an diese Bilder, diese Vor-Bilder einer durchaus unfeierlichen Humanität zu erinnern.

38 Kafka (wie Anm. 37), S. 523.

39 Ebd., S. 522. 
Bereitgestellt von | SUB Göttingen

Angemeldet. | 134.76.162.17

Heruntergeladen am | 07.03.13 13:27 